\title{
Transport process in multi-junctions of quantum systems
}

\author{
Taro Kimura ${ }^{a, 1}$ and Masaki Murata ${ }^{b}$ \\ ${ }^{a}$ Theoretical Research Division, RIKEN Nishina Center, \\ Saitama 351-0198, Japan \\ ${ }^{b}$ Harish-Chandra Research Institute, \\ Chhatnag Road, Jhusi, Allahabad 211019, India \\ E-mail: taro.kimura@riken.jp, m.murata1982@gmail.com
}

ABSTRACT: We consider the junction of multiple one-dimensional systems and study how conserved currents transport at the junction. To characterize the transport process, we introduce reflection/transmission coefficients by applying boundary conformal field theory. We compute the reflection/transmission coefficients for some examples to derive the closed formulas. The formulas demonstrate spin-flip transport, where the spin polarization is flipped at the junction.

KeYwords: Conformal and W Symmetry, Field Theories in Lower Dimensions, Boundary Quantum Field Theory

ARXIV EPRINT: 1505.05275

\footnotetext{
${ }^{1}$ Present address: Department of Physics, Keio University, Kanagawa 223-8521, Japan; E-mail: kimura@phys-h.keio.ac.jp
} 


\section{Contents}

1 Introduction 1

2 Reflection and transmission coefficients 2

2.1 Multi-junction of quantum systems 2

2.2 The R-matrices 4

$\begin{array}{lll}2.3 & \text { Reflection and transmission coefficients } & 6\end{array}$

$\begin{array}{lll}3 & \text { Example I: permutation boundary condition } & 7\end{array}$

4 Example II: coset-type boundary condition $\quad 8$

4.1 Energy transport $\quad 9$

4.2 Current transport 11

$\begin{array}{lll}4.3 & \text { Results } & 12\end{array}$

$\begin{array}{llr}5 & \text { Discussion } & 14\end{array}$

$\begin{array}{ll}\text { A Boundary entropy } & 15\end{array}$

$\begin{array}{ll}\text { B Cardy condition } & 15\end{array}$

\section{Introduction}

Recent development of nanotechnology allows us to build an electric circuit in nanoscale, which involves quantum mechanical nature of electrons. To control such a nanoscale circuit, we need to investigate fundamental properties of quantum wire junctions. Among the theoretical studies of the quantum wire junction, ref. [1] pointed out that the number of connected wires interestingly affects the fixed point of the renormalization group flow. In this sense, the quantum wire junction gains interests not only for engineering applications, but also for fundamental theoretical aspects. For this purpose, there have been a number of works based on conformal field theory (CFT). This is because low-energy behavior of the wider class of one-dimensional systems can be described as Tomonaga-Luttinger liquid (TLL) through the bosonization scheme. See, for example, a textbook on this topic [2].

Most works in this field are based on the TLL description of one-dimensional systems, which is just $c=1$ free boson CFT. The $c=1$ CFT enables us to describe the U(1) degree of freedom, which corresponds to electric charge. However, recent development of spintronics also demands us to incorporate $\mathrm{SU}(2)$ spin degree of freedom into such nanoscale devices. In this case, it is desirable to implement $\mathrm{SU}(2)$ symmetry manifestly in order to investigate the spin-dependent property at the junction. Although the $c=1$ CFT can treat the spin $1 / 2$ system, corresponding to the $\mathrm{SU}(2)_{k=1}$ Kac-Moody algebra, $c \neq 1$ is necessary for 
describing generic spins, due to the identification of the Kac-Moody level $k$ with the spin $s$ as $s=k / 2[3,4]$.

In this paper we study transport process at the multi-junction of one-dimensional systems that have Lie algebraic symmetries. We work with an arbitrary multiplicity $M$ by generalizing the previous works for $M=2[5,6]$. See also [7]. This junction plays a similar role to an impurity in the one-dimensional system. In fact, one can map both the junction system and the impurity system into a $(1+1)$-dimensional system with a boundary by using the folding trick [8-10]. In this picture the information about the junction is implemented into the boundary state for the two dimensional system. Using the boundary state, we shall define the transmission/reflection coefficient of conserved currents at the junction. In addition, to compute the coefficients explicitly, we shall construct a boundary state corresponding to the multi-junction of $\mathrm{SU}(2)$-symmetric systems. We shall compute both energy and spin-current reflection/transmission coefficients to investigate the spindependent property of the junction. In particular the spin transport shows an interesting behavior, namely, the spin-flipping process.

This paper is organized as follows. In section 2, we formulate the transport process at the multi-junction by generalizing the formulation for the multiplicity $M=2[5,6]$. We point out that the R-matrix, which characterizes the transport at the junction, is not symmetric in general for $M>2$, while it is always symmetric when $M=2$. In section 3, we apply this formalism to the permutation boundary condition, which is the simplest example to demonstrate the asymmetric R-matrix. In section 4, we study the transport with the coset-type boundary condition. We shall propose the associated boundary state by generalizing that shown in [11]. The explicit computation of reflection/transmission coefficients shows that the current transport more strongly depends on the multiplicity $M$ than the energy transport. We also discuss its application to the boundary entropy in appendix A. We conclude this paper in section 5 with some discussions.

\section{Reflection and transmission coefficients}

\subsection{Multi-junction of quantum systems}

In this paper, we shall consider the system with $M$ one-dimensional quantum systems connected at a point. Each quantum system is characterized by the following Hamiltonian densities in the field theoretical limit:

$$
\mathcal{H}_{i}=\frac{1}{2 \pi\left(k_{i}+h_{i}^{\vee}\right)} d_{A B}^{i} J^{i, A} J^{i, B}
$$

where $i=1, \ldots, M$ is the label of the quantum systems. $J^{i, A}$ is the current taking values in the Lie algebra $\mathcal{A}_{i}$ and the index $A$ runs over $A=1, \ldots, \operatorname{dim} \mathcal{A}_{i}$. For the moment, we apply generic Lie algebras $\mathcal{A}_{i}$ rather than $s u(2) . d_{A B}^{i}$ is the inverse of the Cartan-Killing form and $h_{i}^{\vee}$ is the dual Coxeter number of the algebra $\mathcal{A}_{i}$. The Fourier modes of the current $J^{i, A}$ satisfy the Kac-Moody algebra $\widehat{\mathcal{A}}_{i}$ :

$$
\left[j_{m}^{i, A}, j_{n}^{i, B}\right]=\left(f^{i}\right)_{C}^{A B} j_{n+m}^{i, C}+k_{i} m d^{i, A B} \delta_{m+n, 0},
$$

where $f^{i}$ is the structure constant of $\mathcal{A}_{i}$ and $k_{i}$ is the level of $\widehat{\mathcal{A}}_{i}$. 

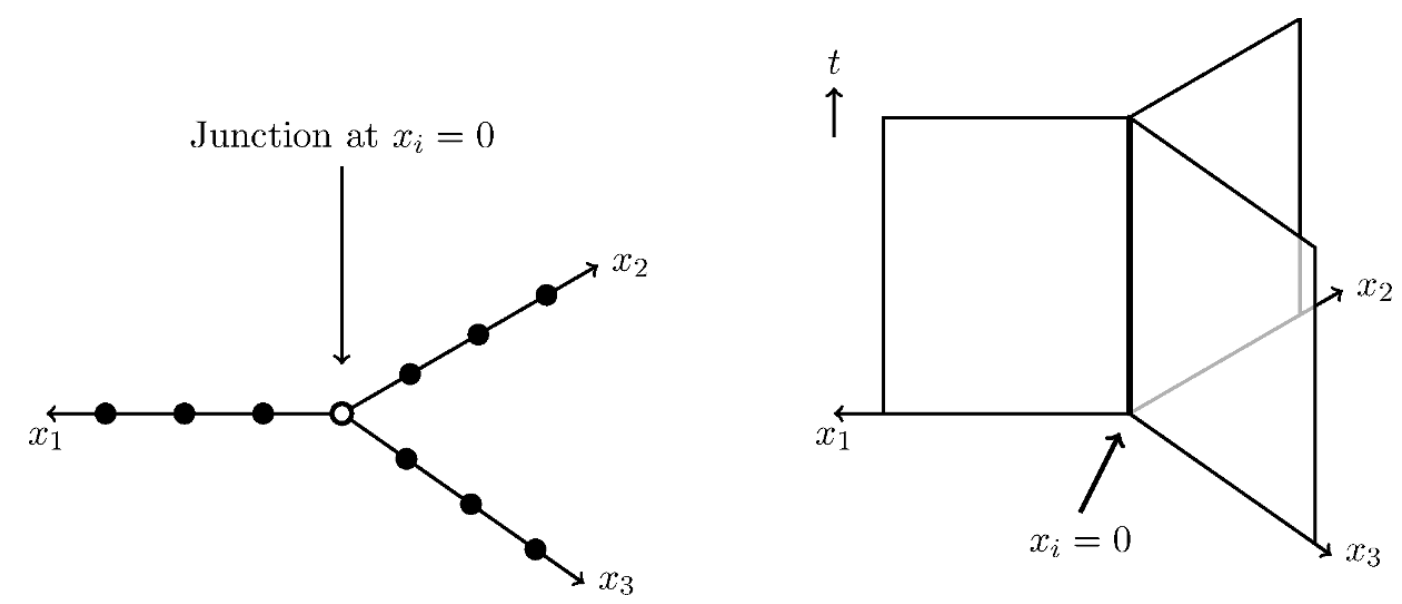

Figure 1. The junction of one-dimensional systems with the multiplicity $M=3$. (Left) Each system is defined through the axis of $x_{i}>0$, and interacting with each other at the sharing origin $x_{i}=0$. (Right) By adding the time direction, one obtains several two-dimensional planes glued along the defect line at $x_{i}=0$.

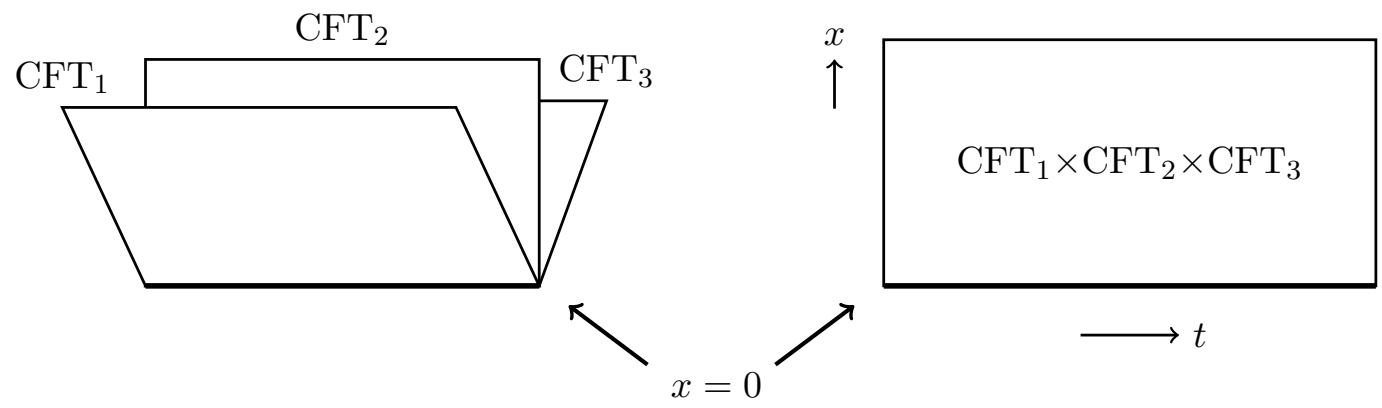

Figure 2. A system with the conformal defect (left) is mapped into another one (right) with the boundary at $x=0$ through the folding trick. For example, a non-trivial bound sate at the junction can be studied using the BCFT approach.

In addition to these Hamiltonians, we also introduce the junction which connects the quantum systems through a local interaction. The interaction occurs if the "spin" $S^{a}$ at the junction takes value in a subalgebra $\mathcal{C}$ of $\mathcal{A}_{i}$. One possible local interaction is described by

$$
\mathcal{H}_{i}^{\mathrm{int}}=\lambda_{i} \delta\left(x_{i}\right) d_{a b} J^{i, a} S^{b},
$$

where $x_{i} \geq 0$ is the coordinate of the quantum systems and the junction is at $x_{i}=0$, as shown in figure 1 . The index $a$ runs as $a=1, \ldots, \operatorname{dim} \mathcal{C}$.

The critical point of this system is $M$-sheeted CFT glued along the conformal defect corresponding to the world line of the junction. This configuration leads to $\mathrm{CFT}_{1} \times$ $\mathrm{CFT}_{2} \times \cdots \times \mathrm{CFT}_{M}$, using the so-called folding trick [8-10]. See figure 2. In the following subsections, we shall define the reflection/transmission coefficient through the boundary CFT (BCFT). The BCFT is characterized by the boundary states $|B\rangle$ which describe the boundary conditions. For example, the energy conservation law along the boundary leads 
to the gluing condition for the Virasoro generators

$$
\left(L_{n}^{\mathrm{tot}}-\bar{L}_{-n}^{\mathrm{tot}}\right)|B\rangle=0
$$

with $L_{n}^{\text {tot }}=\sum_{i=1}^{M} L_{n}^{i}$. On the other hand, the current conservation law needs more consideration. If there is a common subalgebra $\mathcal{C} \subset \mathcal{A}_{i}$ for all $i$, we have

$$
\left(j_{n}^{\text {tot }, a}+\bar{j}_{-n}^{\text {tot }, a}\right)|B\rangle=0
$$

where $j_{n}^{\text {tot, } a}=\sum_{i=1}^{M} j_{n}^{i, a}$ takes value in $\mathcal{C}{ }^{1}$ Furthermore, in general, a subset of $\mathcal{A}_{i}$ 's has a larger subgroup $\mathcal{C}^{\prime} \supset \mathcal{C}$. Supposing that $\mathcal{A}_{i=1, \ldots, l}$ contains $\mathcal{C}^{\prime}$, the gluing condition can be written as

$$
\sum_{i=1}^{l}\left(j_{n}^{i, \alpha}+\bar{j}_{-n}^{i, \alpha}\right)|B\rangle=0
$$

where $j^{i, \alpha}$ takes value in $\mathcal{C}^{\prime} / \mathcal{C}$. On the other hand, if $\mathcal{A}_{i}$ has no bigger common subalgebra with $\mathcal{A}_{j \neq i}$ 's, we have

$$
\left(j_{n}^{i, \mathcal{A}_{i} / C}+\bar{j}_{-n}^{i, \mathcal{A}_{i} / C}\right)|B\rangle=0
$$

Now the properties of the junction are encoded in the boundary state $|B\rangle$.

\subsection{The R-matrices}

To define the reflection/transmission coefficients, we first introduce the R-matrix for the energy by generalizing that for $M=2$,

$$
R_{T}^{i j}=\frac{\left\langle 0\left|L_{2}^{i} \bar{L}_{2}^{j}\right| B\right\rangle}{\langle 0 \mid B\rangle}
$$

Due to the gluing condition for the total current (2.4), the R-matrix satisfies the following constraints

$$
\sum_{i=1}^{M} R_{T}^{i j}=\frac{c_{j}}{2}, \quad \sum_{j=1}^{M} R_{T}^{i j}=\frac{c_{i}}{2} .
$$

These conditions give $2 M-1$ constraints for the matrix elements. As a consequence of these constraints, the R-matrix has $M^{2}-2 M+1=(M-1)^{2}$ degrees of freedom in total. Notice that this reproduces the result of $M=2$, which yields only one degree of freedom [5,6]. Let us introduce another basis to express the $(M-1)^{2}$ degrees of freedom in the R-matrix,

$$
\omega_{T}^{\alpha \beta}=\frac{\left\langle 0\left|\mathcal{W}_{2}^{\alpha} \overline{\mathcal{W}}_{2}^{\beta}\right| B\right\rangle}{\langle 0 \mid B\rangle}
$$

\footnotetext{
${ }^{1}$ This gluing condition is the special case of the generic form

$$
\left(j_{n}^{a}+\Omega\left(\bar{j}_{-n}^{a}\right)\right)|B\rangle=0 .
$$
}

with an automorphism $\Omega$ preserving the energy-momentum tensor. See, for example, [12]. 
where $\alpha, \beta=1, \ldots, M-1$ and

$$
\mathcal{W}_{m}^{\alpha}=\sqrt{\frac{2}{c_{\alpha+1} C_{\alpha} C_{\alpha+1}}}\left(c_{\alpha+1} \sum_{\beta=1}^{\alpha} L_{m}^{\beta}-C_{\alpha} L_{m}^{\alpha+1}\right), \quad C_{\alpha}=\sum_{\beta=1}^{\alpha} c_{\beta} .
$$

It turns out that this $\mathcal{W}_{-2}^{\alpha}|0\rangle$ forms an orthonormal basis,

$$
\left\langle 0\left|\mathcal{W}_{2}^{\alpha} \mathcal{W}_{-2}^{\beta}\right| 0\right\rangle=\delta_{\alpha \beta} \quad \alpha, \beta=1, \ldots, M-1
$$

Then, to express the R-matrix in terms of $\omega_{T}^{\alpha \beta}$, we introduce the inverse transformation of $(2.12)$,

$$
L_{m}^{i}=\sum_{\alpha=1}^{M-1} A_{T}^{i \alpha} \mathcal{W}_{m}^{\alpha}+A_{T}^{i M} L_{m}^{\mathrm{tot}}
$$

with

$$
\begin{aligned}
& A_{T}^{i \alpha}=c_{i} \sqrt{\frac{c_{\alpha+1}}{2 C_{\alpha} C_{\alpha+1}}}, \quad(i \leq \alpha<M), \quad A_{T}^{i+1, i}=-\sqrt{\frac{c_{i+1} C_{i}}{2 C_{i+1}}}, \\
& A_{T}^{i M}=\frac{c_{i}}{C_{M}}, \quad A_{T}^{i \alpha}=0, \quad(i>M+1)
\end{aligned}
$$

Using the coefficients $A$, we get

$$
R_{T}^{i j}=\sum_{\alpha, \beta=1}^{M-1} A_{T}^{i \alpha} A_{T}^{j \beta} \omega_{T}^{\alpha \beta}+\frac{c_{\text {tot }}}{2} A_{T}^{i M} A_{T}^{j M}
$$

where we have used

$$
\left\langle 0\left|\mathcal{W}_{2}^{\alpha} \bar{L}_{2}^{\text {tot }}\right| B\right\rangle=\left\langle 0\left|L_{2}^{\text {tot }} \overline{\mathcal{W}}_{2}^{\alpha}\right| B\right\rangle=0 .
$$

It is worth to emphasize that the R-matrix is not symmetric in general, while it is always symmetric for $M=2$. This is one of the outcomes of the fact that the currents can transmit through more than one channel.

In the same way, we define the R-matrix for the currents $j^{i}$ taking a value in the algebra $\mathcal{A}_{i}$,

$$
R_{J}^{i j, A B}=-\frac{\left\langle 0\left|j_{1}^{i, A} \bar{j}_{1}^{j, B}\right| B\right\rangle}{\langle 0 \mid B\rangle} .
$$

If we restrict to the common subalgebra $\mathcal{C}$, the symmetry guarantees that the R-matrix is in the product form as $R_{J}^{i j, a b}=d^{a b} R_{J}^{i j}$. Without loss of generality, we can assume that no pair of $\mathcal{A}_{i}$ has bigger common subalgebra $\mathcal{C}^{\prime} \supset \mathcal{C}$. If such a $\mathcal{C}^{\prime}$ exists, we can focus on the subsector of the R-matrix associated with $\mathcal{C}^{\prime} / \mathcal{C}$ and do the same procedure as below. With this setup the matrix elements including the index of $\mathcal{A}_{i} / \mathcal{C}$ are

$$
R^{i j, A^{\prime} B^{\prime}}=k_{i} d^{A^{\prime} B^{\prime}} \delta^{i j}, \quad R^{i j, A^{\prime} a}=R^{i j, a A^{\prime}}=0
$$


for any $i, j$. And here $A^{\prime}$ is the index of $\mathcal{A}_{i} / \mathcal{C}$ or $\mathcal{A}_{j} / \mathcal{C}$. On the other hand, the R-matrix for $\mathcal{C}$ satisfies the constraints given by replacing a pair $\left(R_{T}^{i j}, c_{i}\right)$ with $\left(R_{J}^{i j}, 2 k_{i}\right)$ in $(2.10)$,

$$
\sum_{i=1}^{M} R_{J}^{i j}=k_{j}, \quad \sum_{j=1}^{M} R_{J}^{i j}=k_{i} .
$$

We can now utilize the same argument to obtain

$$
R_{J}^{i j}=\sum_{\alpha, \beta=1}^{M-1} A_{J}^{i \alpha} A_{J}^{j \beta} \omega_{J}^{\alpha \beta}+k_{\mathrm{tot}} A_{J}^{i M} A_{J}^{j M}
$$

using the orthonormal basis

$$
\begin{gathered}
\omega_{J}^{\alpha \beta} d^{a b}=-\frac{\left\langle 0\left|\hat{K}_{1}^{\alpha, a} \hat{\hat{K}}_{1}^{\beta, b}\right| B\right\rangle}{\langle 0 \mid B\rangle} \\
\hat{K}_{m}^{\alpha}=\sqrt{\frac{1}{k_{\alpha+1} \kappa_{\alpha} \kappa_{\alpha+1}}} K_{m}^{\alpha}, \quad K_{m}^{\alpha}=\left(k_{\alpha+1} \sum_{\beta=1}^{\alpha} j_{m}^{\beta}-\kappa_{\alpha} j_{m}^{\alpha+1}\right), \quad \kappa_{\alpha}=\sum_{\beta=1}^{\alpha} k_{\beta},
\end{gathered}
$$

with

$$
\begin{aligned}
& A_{J}^{i \alpha}=k_{i} \sqrt{\frac{k_{\alpha+1}}{\kappa_{\alpha} \kappa_{\alpha+1}}}, \quad(i \leq \alpha<M), A_{J}^{i+1, i}=-\sqrt{\frac{k_{i+1} \kappa_{i}}{\kappa_{i+1}}}, \\
& A_{J}^{i M}=\frac{k_{i}}{\kappa_{M}}, \quad A_{J}^{i M}=0, \quad(i>n+1) .
\end{aligned}
$$

\subsection{Reflection and transmission coefficients}

Now we shall define the reflection and transmission coefficients using the R-matrices defined above. As in refs. [5, 6], it is natural to relate the diagonal and off-diagonal elements of the R-matrix to the reflection and transmission coefficients respectively. For the simplest case $M=2[5,6]$, the transmission rate is defined by the "average" of the off-diagonal elements since $R^{12}=R^{21}$, which is derived from the conservation law. However, for $M>2$, the conservation law cannot give such a strong constraint, and thus the average is not suitable to characterize the transport process. Therefore, we set $\mathcal{T}^{i j}$ the transmission coefficient from system $i$ to $j$ with $i \neq j$, and we shall treat $\mathcal{T}^{i j}$ and $\mathcal{T}^{j i}$ as independent variables. Physically it is plausible to demand

$$
1=\mathcal{R}_{T}^{i}+\sum_{j \neq i} \mathcal{T}_{T}^{i j}, \quad 1=\mathcal{R}_{J}^{i}+\sum_{j \neq i} \mathcal{T}_{J}^{i j}, \quad i=1, \ldots, M,
$$

where $\mathcal{R}^{i}$ is the reflection coefficient for the $i$-th system. The constraints (2.10) and (2.21) lead us to define

$$
\begin{aligned}
\mathcal{T}_{T}^{i j} & =\frac{2}{c_{i}} R_{T}^{i j}, & \mathcal{T}_{J}^{i j} & =\frac{1}{k_{i}} R_{J}^{i j}, \\
\mathcal{R}_{T}^{i} & =\frac{2}{c_{i}} R_{T}^{i i}, & \mathcal{R}_{J}^{i} & =\frac{1}{k_{i}} R_{J}^{i i}
\end{aligned}
$$




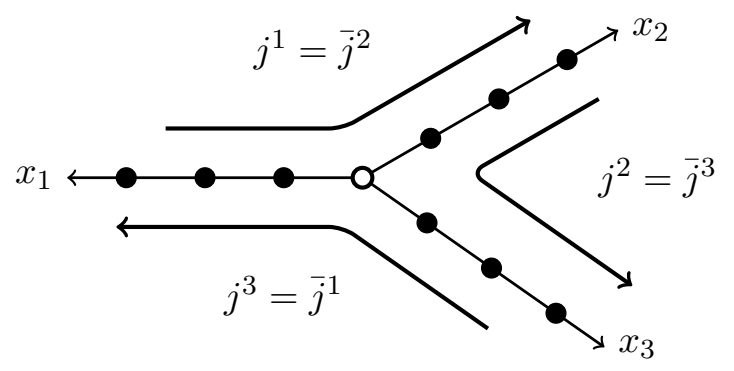

Figure 3. The permutation boundary condition for $M=3$. All the currents for $\mathcal{C}$ are transmitted to the next channel as described by the boundary condition (3.1).

Here $R_{J}^{i j}$ is the R-matrix restricted to the subalgebra $\mathcal{C}$, assuming that no pair of $\mathcal{A}_{i}$ 's have a bigger common subalgebra. For $\mathcal{A}_{i} / \mathcal{C}$, it is plausible to set $\mathcal{R}_{J}^{i}=1$ and $\mathcal{T}_{J}^{i j}=0$ due to the gluing condition (2.8).

This definition does not reduce to the previous ones for $M=2[5,6]$. However, since the R-matrix is symmetric for $M=2$, we have

$$
\mathcal{T}_{T}^{12}=\frac{c_{2}}{c_{1}} \mathcal{T}_{T}^{21}=\frac{c_{1}+c_{2}}{c_{1}} \mathcal{T}_{T}^{\mathrm{avr}}
$$

where $\mathcal{T}_{T}^{\text {avr }}$ is the transmission coefficient defined in [5,6]. The similar relation holds for the current. As stated above, the new definition can be naturally extended to $M>2$.

In the following two sections, we shall compute the reflection/transmission coefficients for two examples.

\section{Example I: permutation boundary condition}

We first consider the case where the boundary condition is given by

$$
J^{i, a}(z)=\bar{J}^{i+1, a}(\bar{z}), \quad J^{i, \mathcal{A}_{i} / \mathcal{C}}(z)=\bar{J}^{i, \mathcal{A}_{i} / \mathcal{C}}(\bar{z})
$$

with $J^{M+1}=J^{1}$ as shown in figure 3 . Here $\mathcal{C}$ is a subalgebra of all the $\mathcal{A}_{i}$. This gluing condition is consistent when all $k_{i}$ have the same value $k$. As stated in the previous section, $\mathcal{R}_{J}^{i}=1$ for $\mathcal{A}_{i} / \mathcal{C}$. For $\mathcal{C}$, we can straightforwardly compute $R_{J}^{i j}$, and non-vanishing components are

$$
R_{J}^{12}=R_{J}^{23}=\cdots=R_{J}^{M 1}=k
$$

This expression shows that the transmission satisfies the cyclic property, which is inherited from the boundary condition (3.1). This is a simple example of the asymmetric R-matrix for $M \geq 3$, while it always becomes symmetric for $M=2$. The transport coefficients for $\mathcal{C}$ are

$$
\mathcal{R}_{J}^{i}=0, \quad \mathcal{T}_{J}^{i j}=\delta_{i, j-1}
$$

This in fact satisfies the constraint (2.27). 
On the other hand, the R-matrix for the energy transport $R_{T}^{i j}$ is more non-trivial,

$$
R_{T}^{i j}=\frac{\left\langle 0\left|L_{2}^{i} \bar{L}_{2}^{j}\right| B\right\rangle}{\langle 0 \mid B\rangle}=\frac{\left\langle 0\left|L_{2}^{i}\left(L_{-2}^{j-1, \mathcal{C}}+L_{-2}^{j, \mathcal{A}_{j} / \mathcal{C}}\right)\right| B\right\rangle}{\langle 0 \mid B\rangle}=\frac{c}{2} \delta_{i, j-1}+\frac{c_{i}-c}{2} \delta_{i j} .
$$

where $c$ is the central charge corresponding to the algebra $\mathcal{C}$ with level $k$. Thus the transport coefficients are

$$
\mathcal{R}_{T}^{i}=1-\frac{c}{c_{i}}, \quad \mathcal{T}_{T}^{i j}=\frac{c}{c_{i}} \delta_{i, j-1} .
$$

Again it is easy to check (2.27). Because $c_{i}>c$, we have $\mathcal{T}_{T}^{i j}<1$. The physical interpretation of $\mathcal{T}_{J}$ and $\mathcal{T}_{T}$ is clear: the currents of $\mathcal{C}$ are completely transmitted, bearing a fraction $c / c_{i}$ of the total energy, while the remaining currents, with a relative energy share of $1-c / c_{i}$, are completely reflected.

\section{Example II: coset-type boundary condition}

Now we shall consider the $M$-junction of $\mathrm{SU}(2)$ spin chains. To be more specific, we consider the cascade of breaking to the diagonal subgroup $\mathrm{SU}(2)_{\kappa_{M}}$ :

$$
\begin{aligned}
& \mathrm{SU}(2)_{k_{1}} \times \mathrm{SU}(2)_{k_{2}} \times \cdots \times \mathrm{SU}(2)_{k_{M}} \\
& \rightarrow \frac{\mathrm{SU}(2)_{k_{1}} \times \mathrm{SU}(2)_{k_{2}}}{\mathrm{SU}(2)_{\kappa_{2}}} \times \frac{\mathrm{SU}(2)_{\kappa_{2}} \times \mathrm{SU}(2)_{k_{3}}}{\mathrm{SU}(2)_{\kappa_{3}}} \times \cdots \times \frac{\mathrm{SU}(2)_{\kappa_{M-1}} \times \mathrm{SU}(2)_{k_{M}}}{\mathrm{SU}(2)_{\kappa_{M}}} \times \mathrm{SU}(2)_{\kappa_{M}} .
\end{aligned}
$$

We claim that the corresponding boundary state is given by the following generalization of $[11]$ :

$$
\left.|B(\rho, r)\rangle=\sum_{\mu, m} \prod_{i=1}^{M} \frac{S_{\rho_{i} \mu_{i}}^{\left(k_{i}\right)}}{\sqrt{S_{0 \mu_{i}}^{\left(k_{i}\right)}}} \prod_{i=1}^{M-1} \frac{S_{r_{i} m_{i}}^{\left(\kappa_{i+1}\right)}}{S_{0 m_{i}}^{\left(\kappa_{i+1}\right)}}|(\mu, m)\rangle\right\rangle
$$

where

$$
\left.\left.\left.\left.|(\mu, m)\rangle\rangle=\left|\mu_{1}, \mu_{2}, m_{1}\right\rangle\right\rangle \otimes\left|m_{1}, \mu_{3}, m_{2}\right\rangle\right\rangle \otimes \cdots \otimes\left|m_{M-2}, \mu_{M}, m_{M-1}\right\rangle\right\rangle \otimes\left|m_{M-1}\right\rangle\right\rangle
$$

is a product of $(M-1)$ Ishibashi states of each coset $\mathrm{SU}(2)_{\kappa_{i}} \times \mathrm{SU}(2)_{k_{i+1}} / \mathrm{SU}(2)_{\kappa_{i+1}}$ and the Ishibashi state of $\mathrm{SU}(2)_{\kappa_{M}}$. In appendix B, we shall show that this boundary state satisfies the Cardy condition. The other constraints on the boundary states, i.e. the sewing relations, are assumed.

The parameters $(\rho, r)$ run over $2 \rho_{i}=0,1, \cdots, k_{i}, 2 r_{i}=0,1, \cdots, \kappa_{i+1}$, and $(\mu, m)$ runs over the same region as $(\rho, r)$ satisfying the additional constraints:

$$
\mu_{1}+\mu_{2}+m_{1} \in \mathbb{Z}, \quad m_{1}+\mu_{3}+m_{2} \in \mathbb{Z}, \quad \cdots, m_{M-2}+\mu_{M}+m_{M-1} \in \mathbb{Z} .
$$

Not all the states labeled by $(\rho, r)$ are independent. This is because the boundary state is invariant under

$$
\rho_{i} \rightarrow J_{i-1,2} \rho_{i}, \quad r_{i} \rightarrow J_{i, 3} J_{i+1,1}^{-1} r_{i}
$$


with $J_{0,2}=J_{1,1}, J_{M, 1}=1$. Here, $\left(J_{i 1}, J_{i 2}, J_{i 3}\right)$ is an element of the identification group of $\mathrm{SU}(2)_{\kappa_{i}} \times \mathrm{SU}(2)_{k_{i+1}} / \mathrm{SU}(2)_{\kappa_{i+1}}$. The two elements of that identification group can be expressed as

$$
\left(J_{i 1} \mu_{1}, J_{i 2} \mu_{2}, J_{i 3} \mu_{3}\right)=\left(\mu_{1}, \mu_{2}, \mu_{3}\right),\left(\kappa_{i} / 2-\mu_{1}, k_{i+1} / 2-\mu_{2}, \kappa_{i+1} / 2-\mu_{3}\right),
$$

where $\left(\mu_{1}, \mu_{2}, \mu_{3}\right)$ labels a primary state of $\mathrm{SU}(2)_{\kappa_{i}} \times \mathrm{SU}(2)_{k_{i+1}} / \mathrm{SU}(2)_{\kappa_{i+1}}$.

\subsection{Energy transport}

Recall that the R-matrix is defined by the overlap between $\langle 0| L_{2}^{i} \bar{L}_{2}^{j}$ and the boundary state $|B\rangle$, which is now written in terms of coset states. The descendant state $L_{-2}^{i}|0\rangle$ shall be expanded by $\mathrm{SU}(2)_{\kappa_{M}}$-singlet states with conformal weight $h=2$,

$$
L_{-2}^{i}|0\rangle=\sum_{A=1}^{M(M+1) / 2} \mathcal{L}_{A}^{i}\left|\Sigma^{A}\right\rangle,
$$

where $\left|\Sigma^{A}\right\rangle$ form a complete set of such singlet states. Here we shall use the explicit form $\left\{\left|\Sigma^{A}\right\rangle\right\}=\left\{|V\rangle,\left|W^{\alpha}\right\rangle,\left|X^{\alpha, \beta}\right\rangle,\left|Y^{\alpha}\right\rangle\right\}$ :

$$
\begin{aligned}
|V\rangle & =|0,0,0\rangle_{1} \otimes \cdots \otimes|0,0,0\rangle_{M-1} \otimes L_{-2}|0\rangle \\
\left|W^{\alpha}\right\rangle & =|0,0,0\rangle_{1} \otimes \cdots \otimes L_{-2}|0,0,0\rangle_{\alpha} \otimes \cdots \otimes|0,0,0\rangle_{M-1} \otimes|0\rangle \\
\left|X^{\alpha, \beta}\right\rangle & =K_{-1}^{\alpha} \cdot \widetilde{K}_{-1}^{\beta}|0\rangle \\
& \propto|0,0,0\rangle_{1} \otimes \cdots \otimes|0,0,0\rangle_{\alpha-1} \otimes|0,0,1\rangle_{\alpha} \otimes|1,0,1\rangle_{\alpha+1} \\
& \quad \otimes \cdots \otimes|1,0,1\rangle_{\beta-1} \otimes|1,0,0\rangle_{\beta} \otimes|0,0,0\rangle_{\beta+1} \otimes \cdots|0,0,0\rangle_{M-1} \otimes|0\rangle \\
\left|Y^{\alpha}\right\rangle & =K_{-1}^{\alpha} \cdot j_{-1}^{\text {tot }}|0\rangle \\
& \propto|0,0,0\rangle_{1} \otimes \cdots \otimes|0,0,0\rangle_{\alpha-1} \otimes|0,0,1\rangle_{\alpha} \otimes|1,0,1\rangle_{\alpha+1} \otimes \cdots \otimes|1,0,1\rangle_{M-1} \otimes|0\rangle
\end{aligned}
$$

with $\widetilde{K}_{n}^{\alpha, a}=k_{\alpha+1} \sum_{\beta=1}^{\alpha} j_{n}^{\beta, a}-\left(\kappa_{\alpha}+4\right) j_{n}^{\alpha+1, a}$. Here the indices run as $\alpha, \beta=1, \ldots, M-1$ with $\alpha<\beta$. Thus the total number of the basis is given by $1+(M-1)+(M-1)(M-$ $2) / 2+(M-1)=M(M+1) / 2$.

Since the boundary state only has diagonal supports, we have

$$
\begin{aligned}
R_{T}^{i j} & =\sum_{A=1}^{M(M+1) / 2} \mathcal{L}_{A}^{i} \mathcal{L}_{A}^{j} \frac{\left\langle\Sigma^{A}\right| \otimes\left\langle\Sigma^{A} \mid B\right\rangle}{\langle 0 \mid B\rangle} \\
& =\sum_{A=1}^{M(M+1) / 2} \frac{L_{C}\left(i, \Sigma^{A}\right) L_{C}\left(j, \Sigma^{A}\right)}{\left\langle\Sigma^{A} \mid \Sigma^{A}\right\rangle} \frac{\left\langle\sigma^{A}\right| \otimes\left\langle\sigma^{A} \mid B\right\rangle}{\langle 0 \mid B\rangle},
\end{aligned}
$$

with the normalized state $\left|\sigma^{A}\right\rangle=\left|\Sigma^{A}\right\rangle / \sqrt{\left\langle\Sigma^{A} \mid \Sigma^{A}\right\rangle}$ and

$$
L_{C}\left(i, \Sigma^{A}\right)=\left\langle\Sigma^{A}\left|L_{-2}^{i}\right| 0\right\rangle=\mathcal{L}_{A}^{i}\left\langle\Sigma^{A} \mid \Sigma^{A}\right\rangle .
$$

This expression (4.9) shows $R_{T}^{i j}=R_{T}^{j i}$; the boundary state (4.2) gives the symmetric Rmatrix for energy. The symmetry of the R-matrix may be understood as follows. Recall 
that our boundary state corresponds to the cascade of breaking (4.1). At each step of the cascade we have the diagonal coset of $\mathrm{SU}(2)$, which gives the symmetric R-matrix $[5,6]$. Since all steps give the symmetric R-matrix, the final form of the R-matrix is symmetric. In the next subsection, we shall see that the spin-current R-matrix is also symmetric.

From now we shall compute $L_{C},\left\langle\Sigma^{A} \mid \Sigma^{A}\right\rangle$ and $\left\langle\sigma^{A}\right| \otimes\left\langle\sigma^{A} \mid B\right\rangle /\langle 0 \mid B\rangle$ one by one. The straightforward computation gives $L_{C}$ in the form of

$$
\begin{aligned}
L_{C}(i, V) & =\frac{3 k_{i}}{2\left(\kappa_{M}+2\right)}, \\
L_{C}\left(i, W^{\alpha}\right) & =\frac{3 k_{i} k_{\alpha+1}}{2\left(\kappa_{\alpha}+2\right)\left(\kappa_{\alpha+1}+2\right)} U(\alpha-i)+\frac{3 k_{i} \kappa_{i-1}}{2\left(k_{i}+2\right)\left(\kappa_{i}+2\right)} \delta_{\alpha+1, i}, \\
L_{C}\left(i, X^{\alpha, \beta}\right) & =\frac{3}{2} k_{\alpha+1} k_{\beta+1} k_{i} U(\alpha-i)-\frac{3}{2} \kappa_{\alpha} k_{\beta+1} k_{i} \delta_{i, \alpha+1}, \\
L_{C}\left(i, Y^{\alpha}\right) & =\frac{3 k_{i} k_{\alpha+1}}{2} U(\alpha-i)-\frac{3 k_{i} \kappa_{i-1}}{2} \delta_{i, \alpha+1}
\end{aligned}
$$

where $U(x)$ is a unit step function: $U(x \geq 0)=1, U(x<0)=0$. We can also compute their norms as

$$
\begin{aligned}
\langle V \mid V\rangle & =\frac{c_{\kappa_{M}}}{2}, \\
\left\langle W^{\alpha} \mid W^{\alpha}\right\rangle & =\frac{c_{\kappa_{\alpha}}+c_{\alpha+1}-c_{\kappa_{\alpha+1}}}{2}, \\
\left\langle X^{\alpha, \beta} \mid X^{\alpha, \beta}\right\rangle & =\frac{3}{4} k_{\alpha+1} k_{\beta+1} \kappa_{\alpha} \kappa_{\alpha+1}\left(k_{\beta+1} \kappa_{\beta}+\left(\kappa_{\beta}+4\right)^{2}+4 k_{\beta+1}\right), \\
\left\langle Y^{\alpha} \mid Y^{\alpha}\right\rangle & =\frac{3}{4} \kappa_{\alpha} \kappa_{\alpha+1} k_{\alpha+1}\left(\kappa_{M}+4\right) .
\end{aligned}
$$

where $c_{i}$ and $c_{\kappa_{i}}$ are the central charges of $\mathrm{SU}(2)_{k_{i}}$ and $\mathrm{SU}(2)_{\kappa_{i}}$ respectively.

Then we have the overlaps between the boundary state (4.2) and the normalized states $\left\{\left|\sigma^{A}\right\rangle\right\}=\left\{|v\rangle,\left|w^{\alpha}\right\rangle,\left|x^{\alpha, \beta}\right\rangle,\left|y^{\alpha}\right\rangle\right\}$

$$
\begin{aligned}
\frac{\langle v| \otimes\langle v \mid B\rangle}{\langle 0 \mid B\rangle} & =1, \\
\frac{\left\langle w^{\alpha}\right| \otimes\left\langle w^{\alpha} \mid B\right\rangle}{\langle 0 \mid B\rangle} & =1, \\
\frac{\left\langle x^{\alpha, \beta}\right| \otimes\left\langle x^{\alpha, \beta} \mid B\right\rangle}{\langle 0 \mid B\rangle} & =\left(\prod_{i=\alpha}^{\beta-1} \frac{S_{r_{i} 1}^{\kappa_{i+1}} S_{00}^{\kappa_{i+1}}}{S_{01}^{\kappa_{i+1}} S_{r_{i} 0}^{\kappa_{i+1}}}\right), \\
\frac{\left\langle y^{\alpha}\right| \otimes\left\langle y^{\alpha} \mid B\right\rangle}{\langle 0 \mid B\rangle} & =\left(\prod_{i=\alpha}^{M-1} \frac{S_{r_{i} 1}^{\kappa_{i+1}} S_{00}^{\kappa_{i+1}}}{S_{01}^{\kappa_{i+1}} S_{r_{i} 0}^{\kappa_{i+1}}}\right) .
\end{aligned}
$$

Inputting above data into (4.9), we obtain the closed expression of the R-matrix. It is intriguing that the result is independent of $\rho_{i}$, which was also for $M=2[5,6]$. As in the case of the Kondo problem, the parameter $r_{i}$ could be interpreted as the effective spin at the junction [13]. This result implies the energy transport is basically characterized by this residual effective spin of the junction. As we will see below, this property is also observed for the current transport. 


\subsection{Current transport}

Let us now compute the reflection and transmission coefficients for Kac-Moody current with the boundary state (4.2). As shown below, the computation for the current is actually simpler than that for the energy. To compute the $\omega_{J}^{\alpha \beta}(2.23)$, it turns out to be helpful to write $\widehat{K}_{-1}^{\alpha,+}|0\rangle$ in terms of the coset states. $\widehat{K}_{-1}^{\alpha,+}$ gives the descendants of $\mathrm{SU}(2)_{\kappa_{\alpha}}$ and $\mathrm{SU}(2)_{k_{\alpha+1}}$, and makes the spin 1 state of $\mathrm{SU}(2)_{\kappa_{\alpha+1}}$. Thus we have

$$
\widehat{K}_{-1}^{\alpha,+}|0\rangle=|(\mu, m)\rangle \text { with } \mu_{i}=0, \quad m_{i<\alpha}=0, \quad m_{i \geq \alpha}=1 .
$$

The relevant states to compute $R_{J}$ are the conformal vacuum $|0\rangle$ and the states with the conformal weight $h=\bar{h}=1$ and with spin 1 under $\mathrm{SU}(2)_{\kappa_{M}}$. From (4.2), these contributions are given by

$$
|B(\rho, r)\rangle=W_{M}|0\rangle+\sum_{\alpha=1}^{M-1} W_{\alpha} \widehat{K}_{-1}^{\alpha,+}|0\rangle \widehat{\widehat{K}_{-1}^{\alpha,-}|0\rangle}+\cdots
$$

with

$$
W_{j}=\left(\prod_{i=1}^{M} \frac{S_{\rho_{i} 0}^{\left(k_{i}\right)}}{\sqrt{S_{00}^{\left(k_{i}\right)}}}\right)\left(\prod_{i=1}^{j-1} \frac{S_{r_{i} 0}^{\left(\kappa_{i+1}\right)}}{S_{00}^{\left(\kappa_{i+1}\right)}}\right)\left(\prod_{i=j}^{M-1} \frac{S_{r_{i} 1}^{\left(\kappa_{i+1}\right)}}{S_{01}^{\left(\kappa_{i+1}\right)}}\right) .
$$

Do not confuse this coefficient $W_{j}$ with the singlet state $\left|W^{\alpha}\right\rangle$ which appeared in section 4.1. This leads to

$$
\omega_{J}^{\alpha \beta}=\delta_{\alpha, \beta} \frac{W_{\alpha}}{W_{M}}=\delta_{\alpha, \beta} \prod_{i=\alpha}^{M-1} \frac{S_{00}^{\left(\kappa_{i+1}\right)} S_{r_{i} 1}^{\left(\kappa_{i+1}\right)}}{S_{r_{i} 0}^{\left(\kappa_{i+1}\right)} S_{01}^{\left(\kappa_{i+1}\right)}}
$$

and the R-matrix for the current

$$
\begin{aligned}
& R_{J}^{i j}=k_{i} k_{j}\left(-\frac{1}{K_{\alpha}} \frac{W_{\alpha-1}}{W_{M}}+\sum_{\beta=\alpha}^{M-1} \frac{k_{\beta+1}}{K_{\beta} K_{\beta+1}} \frac{W_{\beta}}{W_{M}}+\frac{1}{K_{M}}\right) \quad(\alpha=\max (i, j)), \\
& R_{J}^{i i}=\frac{k_{i} K_{i-1}}{K_{i}} \frac{W_{i-1}}{W_{M}}+\sum_{\beta=i}^{M-1} \frac{k_{i}^{2} k_{\beta+1}}{K_{\beta} K_{\beta+1}} \frac{W_{\beta}}{W_{M}}+\frac{k_{i}^{2}}{K_{M}} .
\end{aligned}
$$

This expression shows that the spin-current R-matrix is symmetric and is independent of $\rho$ 's as the energy R-matrix. Finally, the reflection and transmission coefficients for the currents are

$$
\begin{aligned}
& \mathcal{T}_{J}^{i j}=-\frac{k_{j}}{K_{\alpha}} \frac{W_{\alpha-1}}{W_{M}}+\sum_{\beta=\alpha}^{M-1} \frac{k_{j} k_{\beta+1}}{K_{\beta} K_{\beta+1}} \frac{W_{\beta}}{W_{M}}+\frac{k_{j}}{K_{M}} \quad(\alpha=\max (i, j)), \\
& \mathcal{R}_{J}^{i}=\frac{K_{i-1}}{K_{i}} \frac{W_{i-1}}{W_{M}}+\sum_{\beta=i}^{M-1} \frac{k_{i} k_{\beta+1}}{K_{\beta} K_{\beta+1}} \frac{W_{\beta}}{W_{M}}+\frac{k_{i}}{K_{M}} .
\end{aligned}
$$

It is straightforward to show the conservation law:

$$
\sum_{j(\neq i)}^{M} \mathcal{T}_{J}^{i j}+\mathcal{R}_{J}^{i}=1
$$



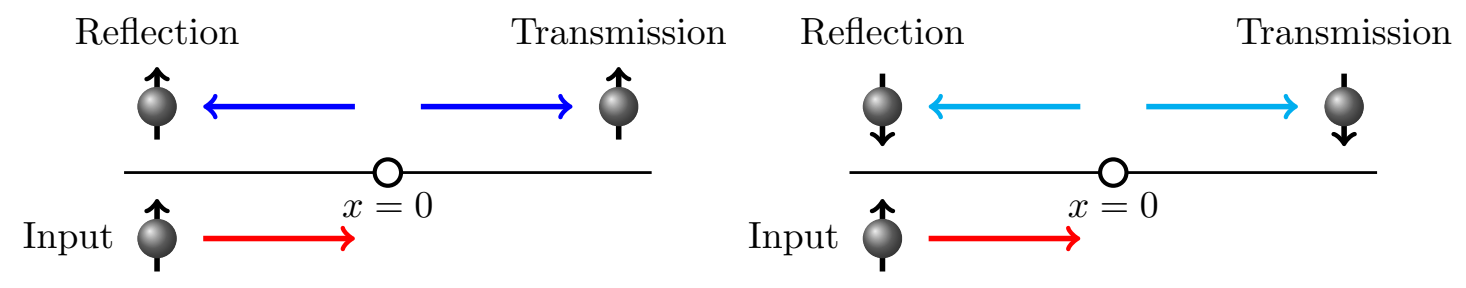

Figure 4. Schematic illustration of current transport process at the defect: (left) non-flipping and (right) spin-flipping processes.

\subsection{Results}

From now on, we shall show results for some multiplicity $M$ and parameters $r_{i}$. First of all, let us check that our formula reproduces the previous results for the simplest case $M=2$. In this case, the Virasoro singlet states are given by $\left\{\left|\Sigma^{A}\right\rangle\right\}=\left\{|V\rangle,\left|W^{1}\right\rangle,\left|Y^{1}\right\rangle\right\}$, and thus we obtain

$$
\begin{aligned}
R_{T}^{i j}= & \frac{L_{C}(i, V) L_{C}(j, V)}{\langle V \mid V\rangle} \frac{\langle v| \otimes\langle v \mid B\rangle}{\langle 0 \mid B\rangle}+\frac{L_{C}\left(i, W^{1}\right) L_{C}\left(j, W^{1}\right)}{\left\langle W^{1} \mid W^{1}\right\rangle} \frac{\left\langle w^{1}\right| \otimes\left\langle w^{1} \mid B\right\rangle}{\langle 0 \mid B\rangle} \\
& +\frac{L_{C}\left(i, Y^{1}\right) L_{C}\left(j, Y^{1}\right)}{\left\langle Y^{1} \mid Y^{1}\right\rangle} \frac{\left\langle y^{1}\right| \otimes\left\langle y^{1} \mid B\right\rangle}{\langle 0 \mid B\rangle} .
\end{aligned}
$$

By substituting (4.11)-(4.13), we reproduce the result of Quella, Runnel and Watts [5] up to the conventions; $r_{1}$ in this paper is $\rho$ in [5]. In a similar way, for the current transport, the formulas (4.18) and (4.19) reproduce our previous result in [6]. Note that, as addressed in section 2.3, the definitions of reflection/transmission coefficients are different, while $R_{J}$ and $R_{T}$ are the same as the former definitions.

The numerical computation with the new definition involves table 1 for $M=2$. The matrix, which we call the reflection/transmission matrix, in the table is defined by

$$
U_{T / J}^{i j}=\left\{\begin{array}{ll}
\mathcal{R}_{T / J}^{i} & (\text { for } i=j) \\
\mathcal{T}_{T / J}^{i j} & (\text { for } i \neq j)
\end{array} .\right.
$$

Here we obtain negative transport coefficients for the current in general. For example, if we apply the gluing condition (2.6) with the automorphism $\Omega=-1$

$$
\Omega\left(\bar{j}_{-n}^{a}\right)=-\bar{j}_{-n}^{a},
$$

we immediately obtain the negative current transport coefficient. This automorphism (4.25) implies redefinition of generators of the corresponding Lie algebra. In particular, for $\mathrm{SU}(2)$, the redefinition of the generator $\sigma_{z} \rightarrow-\sigma_{z}$ means the $z$-spin flip. Therefore the negative transport can be understood as the flipping of the spin polarization at the junction, as shown in figure 4.

We have observed the negative reflection coefficients even for $M=2$ when $k_{1}+k_{2} \geq 4$. Due to the conservation law, not both of the transmission and the reflection coefficients can be negative. Our computation for $1 \leq k_{1}, k_{2} \leq 10$ shows that the transmission coefficients 


\begin{tabular}{|ccc|}
\hline$\left(k_{1}, k_{2}, r_{1}\right)$ & $U_{T}$ & $U_{J}$ \\
\hline$(1,3,1)$ & $\left(\begin{array}{ll}0.15625 & 0.84375 \\
0.46875 & 0.53125\end{array}\right)$ & $\left(\begin{array}{cc}-0.125 & 1.125 \\
0.375 & 0.625\end{array}\right)$ \\
$(1,5,3 / 2)$ & $\left(\begin{array}{ll}0.292893 & 0.707107 \\
0.329983 & 0.670017\end{array}\right)$ & $\left(\begin{array}{cc}-0.178511 & 1.17851 \\
0.235702 & 0.764298\end{array}\right)$ \\
\hline
\end{tabular}

Table 1. Spin-flipping reflection coefficients for $M=2 . U_{T / J}$ is the reflection/transmission matrix defined in (4.24).

\begin{tabular}{|c|c|c|c|c|c|}
\hline$\left(r_{1}, r_{2}, r_{3}\right)$ & $U_{T}$ & \multicolumn{4}{|c|}{$U_{J}$} \\
\hline \multirow{4}{*}{$(0,0,1)$} & $\left.\begin{array}{llll}0.15625 & 0.28125 & 0.28125 & 0.28125\end{array}\right)$ & -0.125 & 0.375 & 0.375 & 0.375 \\
\hline & $\begin{array}{llll}0.28125 & 0.15625 & 0.28125 & 0.28125\end{array}$ & 0.375 & -0.125 & 0.375 & 0.375 \\
\hline & $\begin{array}{llll}0.28125 & 0.28125 & 0.15625 & 0.28125\end{array}$ & 0.375 & 0.375 & -0.125 & 0.375 \\
\hline & $\begin{array}{llll}0.28125 & 0.28125 & 0.28125 & 0.15625\end{array}$ & 0.375 & 0.375 & 0.375 & -0.125 \\
\hline \multirow{4}{*}{$(1 / 2,0,1)$} & $\left.\begin{array}{llll}0.28125 & 0.15625 & 0.28125 & 0.28125\end{array}\right)$ & 0.375 & -0.125 & 0.375 & 0.375 \\
\hline & $\begin{array}{llll}0.15625 & 0.28125 & 0.28125 & 0.28125\end{array}$ & -0.125 & 0.375 & 0.375 & 0.375 \\
\hline & $\begin{array}{llll}0.28125 & 0.28125 & 0.15625 & 0.28125\end{array}$ & 0.375 & 0.375 & -0.125 & 0.375 \\
\hline & $\begin{array}{llll}0.28125 & 0.28125 & 0.28125 & 0.15625\end{array}$ & 0.375 & 0.375 & 0.375 & -0.125 \\
\hline
\end{tabular}

Table 2. Spin-flipping reflection/transmission coefficients for $M=4$ with $k_{i}=1$ for $i=1, \ldots, 4$, corresponding to the quadruple junction of the $s=1 / 2$ Heisenberg spin chains.

are always positive. In addition, keeping $k_{1}=1$ fixed, the large $k_{2}$ gives the large absolute value of the negative reflection coefficient. This implies that the spin chain with higher spins can flip a spin more efficiently. This property would be helpful for actual applications to control the spin current.

For $M>3$, we obtain many examples of the spin-flipping process. In particular, for $M=4$, the spin-flipping is observed for $k_{i}=1$ with $i=1, \ldots, 4$, which is the quadruple junction of the $s=1 / 2$ Heisenberg spin chains, as shown in table 2 .

In contrast to the current transport, it is expected that the energy transport is always (semi-)positive, because it is not possible to provide a reasonable interpretation for the negative energy transport. Up to now, we do not know how to prove this for generic parameters. Even for $M=2$, the complete proof is missing in spite of an attempt given in [5]. For this reason, instead of giving a generic proof, we have done the numerical tests for some values of parameters $M, r_{i}$. For $M=2, k_{i} \leq 10, M=3, k_{i} \leq 5$ and $M=4, k_{i} \leq 2$, we have explicitly observed the semi-positivity of the elements of $U_{T}$. 


\section{Discussion}

In this paper we have discussed the transport process at the multi-junction with respect to both of energy and current flows. We have defined the transport coefficients with arbitrary multiplicity $M$ by modifying and generalizing the previous one for $M=2$. We have applied this formalism to some examples. The permutation boundary condition gives a simple, but important example such that the transport process becomes asymmetric between the channels, which cannot occur in the situation with $M=2$. We have also considered the coset-type boundary condition, in order to study the spin-dependent transport at the junction. Proposing the corresponding boundary state, we have seen the multiplicitydependence of the transport coefficients. By increasing the multiplicity, we have obtained more examples with the negative current transport, while the energy transport coefficients are always positive. This behavior suggests the spin-flip at the junction, which is more specific to high-multiplicity. In particular, for $M=4$, we have observed the spin-flipping reflection and transmission even with the junction of the $s=1 / 2$ Heisenberg spin chains. Thus the quadruple junction of $s=1 / 2$ Heisenberg spin chains seems accessible both in experiments and theoretical studies.

Although the BCFT approach suggests a non-trivial fixed point involving spin-flipping phenomena, it is still unclear what microscopic interaction at the junction induces this phenomena. In addition, the physical meaning of $\rho, r$ is not yet obvious. As mentioned in section 4.1, a plausible interpretation of $r_{i}$ is the effective spin at the junction, and this interpretation is actually applied to the Kondo problem. To understand them, it is important to analyze the multi-junction using another method, e.g. Bethe ansatz. In addition, for engineering applications, it is also required for manipulating the boundary state. A possible direction is to study the relation between the boundary state and the interaction at the junction. There are various choices of interaction terms; (2.3) is not an unique choice. A different interaction term may lead to a different boundary state. To understand how these interactions determine the boundary states is important not only for actual applications, but also for theoretical interests.

In addition to the boundary states used in this paper, there are a number of solutions to the boundary condition. For the corresponding boundary states, it is interesting to study the transport coefficients. For example, Fredenhagen and Quella [14] proposed a new type of boundary states, which is a generalization of the permutation boundary state, used in section 3. However, the form of the boundary states is not well known. One way to find them is to solve string field theory with $\mathrm{SU}(2)_{k_{1}} \times \mathrm{SU}(2)_{k_{2}}$ symmetry. As a first step, the authors are solving string field theory with the single $\mathrm{SU}(2)_{k}$ with collaborators.

\section{Acknowledgements}

MM thanks Ashoke Sen for useful discussions. The work of TK is supported in part by Grant-in-Aid for JSPS Fellows (\#25-4302). The work of MM is supported by JSPS postdoctoral fellowship for research abroad (\#27-14). 


\section{A Boundary entropy}

In addition to the transport process discussed in the main part of this paper, another interesting application of the boundary state is the boundary entropy, which is also called the $g$-factor [15]. The boundary entropy is obtained by the inner product of the boundary state and the conformal vacuum, with a proper subtraction of the "bulk" contribution,

$$
S_{\text {bdry }}=\ln \langle 0 \mid B\rangle-\ln \left\langle 0 \mid B_{0}\right\rangle \text {. }
$$

This bulk contribution $S_{0}=\ln \left\langle 0 \mid B_{0}\right\rangle$ corresponds to the situation in the absence of the interaction between the junction and the bulk. Therefore $\left|B_{0}\right\rangle$ is given by

$$
\left|B_{0}\right\rangle=|0\rangle^{\otimes M}
$$

where $|0\rangle$ 's are Cardy's boundary states for $\mathrm{SU}(2)_{k_{m}}$ for $m=1, \ldots, M$.

As pointed out in the previous work [6], $|0\rangle^{\otimes M}$ is obtained by setting all the parameters to be zero in the boundary state (4.2),

$$
\left|B_{0}\right\rangle=|B(0,0)\rangle \text {. }
$$

Since the overlap between the boundary state and the conformal vacuum is given by

$$
\langle 0 \mid B(\rho, r)\rangle=\prod_{i=1}^{M} \frac{S_{\rho_{i} 0}^{\left(k_{i}\right)}}{\sqrt{S_{00}^{\left(k_{i}\right)}}} \prod_{i=1}^{M-1} \frac{S_{r_{i} 0}^{\left(\kappa_{i+1}\right)}}{\sqrt{S_{00}^{\left(\kappa_{i+1}\right)}}},
$$

we obtain the boundary entropy as follows,

$$
W_{\text {bdry }} \equiv \exp \left(S_{\text {bdry }}\right)=\prod_{i=1}^{M} \frac{S_{\rho_{i} 0}^{\left(k_{i}\right)}}{S_{00}^{\left(k_{i}\right)}} \prod_{i=1}^{M-1} \frac{S_{r_{i} 0}^{\left(\kappa_{i+1}\right)}}{S_{00}^{\left(\kappa_{i+1}\right)}} .
$$

Here $W_{\text {bdry }}$ implies the ground state degeneracy for the boundary, which is referred to the $g$-factor. This expression can be directly applied to the spin-chain junction under the identification of the Kac-Moody level $k_{i}$ with the spin representation $s_{i}$ as $s_{i}=k_{i} / 2[3,4]$. It is interesting to check the formula (A.5) by studying the discrete lattice models with the Bethe ansatz method.

\section{B Cardy condition}

Boundary states should satisfy consistency relations: the Cardy condition and the sewing relations. In this appendix, we shall show that (4.2) satisfies the Cardy condition. While we have considered $M$ products of SU(2) in section 4, we shall treat $M$ products of a generic group $G$ in this appendix. Now $\rho_{i}$ and $r_{i}$ are the weight of $G_{k_{i}}$ and $G_{\kappa_{i}}$ respectively. In the same way, the region of $(\mu, m)$ is specified by $\mathrm{All}_{G}$ (see eq. (12) of ref. [11])

$$
(\mu, m) \in \operatorname{All}_{G} \Leftrightarrow \mu_{1}+\mu_{2}-m_{1} \in \mathcal{G}, m_{1}+\mu_{3}-m_{2} \in \mathcal{G}, \cdots, m_{M-2}+\mu_{M}-m_{M-1} \in \mathcal{G}
$$


with $\mathcal{G}$ the root lattice of $G$. This reduces to (4.4) when $G=\mathrm{SU}(2)$. Notice that the projection operator, which appears in [11], is trivial in this case.

Now let us compute the partition function on the cylinder,

$$
\begin{aligned}
& Z_{(\rho, r),(\tau, t)}=\left\langle\rho, r\left|\widetilde{q}^{L_{0}+\bar{L}_{0}-c / 12}\right| \tau, t\right\rangle \\
& =\sum_{(\mu, m) \in \operatorname{All}_{G}}\left(\prod_{i=1}^{M} \frac{\bar{S}_{\rho_{i} \mu_{i}}^{k_{i}}}{\sqrt{S_{0 \mu_{i}}^{k_{i}}}}\right)\left(\prod_{i=1}^{M-1} \frac{S_{r_{i} m_{i}}^{\kappa_{i+1}}}{S_{0 m_{i}}^{\kappa_{i+1}}}\right)\left(\prod_{i=1}^{M} \frac{S_{\tau_{i} \mu_{i}}^{k_{i}}}{\sqrt{S_{0 \mu_{i}}^{k_{i}}}}\right)\left(\prod_{i=1}^{M-1} \frac{\bar{S}_{t_{i} m_{i}}^{\kappa_{i+1}}}{S_{0 m_{i}}^{\kappa_{i+1}}}\right) \\
& \times \widetilde{\chi}_{\mu_{1}, \mu_{2}, m_{1}} \widetilde{\chi}_{m_{1}, \mu_{3}, m_{2}} \cdots \widetilde{\chi}_{m_{M-2}, \mu_{M}, m_{M-1}} \widetilde{\chi}_{m_{M-1}}
\end{aligned}
$$

where $\tilde{\chi}=\chi(\widetilde{q})$. Using the modular S-matrix, we get

$$
\begin{aligned}
& Z_{(\rho, r),(\tau, t)}=\left|\mathcal{G}_{\mathrm{id}}\right|^{M-1} \sum_{\substack{(\mu, m) \in \mathrm{All}_{G} \\
[\nu, n, p] \in \operatorname{Rep}_{G}}}\left(\prod_{i=1}^{M} \frac{\bar{S}_{\rho_{i} \mu_{i}}^{k_{i}} S_{\tau_{i} \mu_{i}}^{k_{i}}}{S_{0 \mu_{i}}^{k_{i}}}\right)\left(\prod_{i=1}^{M-1} \frac{S_{r_{i} m_{i}}^{\kappa_{i+1}} \bar{S}_{t_{i} m_{i}}^{\kappa_{i+1}}}{S_{0 m_{i}}^{\kappa_{i+1}} S_{0 m_{i}}^{\kappa_{i}+1}}\right) \\
& \times\left(\prod_{i=1}^{M} S_{\mu_{i} \nu_{i}}^{k_{i}}\right)\left(\prod_{i=1}^{M-1} S_{m_{i} n_{i}}^{\kappa_{i+1}} S_{m_{i} p_{i}}^{\kappa_{i+1}}\right) \chi_{\nu_{1}, \nu_{2}, n_{1}} \chi_{p_{1}, \nu_{3}, n_{2}} \cdots \chi_{p_{M-2}, \nu_{M}, n_{M-1}} \chi_{p_{M-1}} \\
& =\left|\mathcal{G}_{\text {id }}\right|^{M-1} \sum_{\substack{(\mu, m) \in \operatorname{All}_{G} \\
[\nu, n, p] \in \operatorname{Rep}_{G}}}\left(\prod_{i=1}^{M} \frac{\bar{S}_{\rho_{i} \mu_{i}}^{k_{i}} S_{\tau_{i} \mu_{i}}^{k_{i}} S_{\mu_{i} \nu_{i}}^{k_{i}}}{S_{0 \mu_{i}}^{k_{i}}}\right)
\end{aligned}
$$

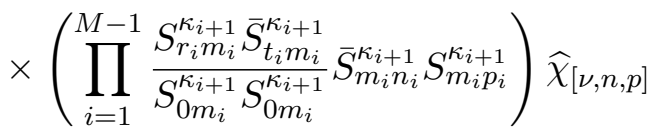

where $\chi=\chi(q)$ and we have used the modular transformation:

$$
\widetilde{\chi}_{m, n, p}=\left|\mathcal{G}_{\text {id }}\right| \sum_{\left(m^{\prime}, n^{\prime}, p^{\prime}\right) \in \operatorname{Rep}_{G}} S_{m m^{\prime}}^{k_{1}} S_{n n^{\prime}}^{k_{2}} \bar{S}_{p p^{\prime}}^{\kappa_{1}} \chi_{m^{\prime} n^{\prime} p^{\prime}}
$$

and $\widehat{\chi}_{[\nu, n, p]}=\chi_{\nu_{1}, \nu_{2}, n_{1}} \chi_{p_{1}, \nu_{3}, n_{2}} \cdots \chi_{p_{M-2}, \nu_{M}, n_{M-1}} \chi_{p_{M-1}} \cdot \mathcal{G}_{\text {id }}$ is the identification group of $G \times G / G$ and $\left|\mathcal{G}_{\text {id }}\right|$ is its dimension. $\operatorname{Rep}_{G}$ is obtained by taking the quotient with respect to $\mathcal{G}_{\text {id }}$ in each coset. For example,

$$
\left(\nu_{1}, \nu_{2}, n_{1}\right) \sim\left(J_{11} \nu_{1}, J_{12} \nu_{2}, J_{13} n_{1}\right)
$$

where $\left(J_{11}, J_{12}, J_{13}\right) \in \mathcal{G}_{\text {id }}$. Now we can use the identity

$$
\begin{aligned}
1=\frac{1}{\left|\mathcal{G}_{\text {id }}\right|^{M-1}} \sum_{J} e^{2 \pi i\left(Q_{J_{11}}\left(\mu_{1}\right)+Q_{J_{12}}\left(\mu_{2}\right)-Q_{J_{13}}\left(m_{1}\right)\right)} & \\
& \times\left(\prod_{i=2}^{M-1} e^{2 \pi i\left(Q_{J_{i 1}}\left(m_{i-1}\right)+Q_{J_{i 2}}\left(\mu_{i+1}\right)-Q_{J_{i 3}}\left(m_{i}\right)\right)}\right)
\end{aligned}
$$


This holds if and only if $(\mu, m) \in \operatorname{Rep}_{G}$ otherwise the right hand side vanishes. By substituting this we get

$$
\begin{aligned}
& Z_{(\mu, r),(\tau, t)}=\sum_{J, d} \sum_{\substack{\mu_{i}, m_{i} \in \operatorname{Rep}(G) \\
[\nu, n, p] \in \operatorname{Rep}_{G}}} e^{2 \pi i\left(Q_{J_{11}}\left(\mu_{1}\right)+Q_{J_{12}}\left(\mu_{2}\right)-Q_{J_{13}}\left(m_{1}\right)\right)} \\
& \times\left(\prod_{i=2}^{M-1} e^{2 \pi i\left(Q_{J_{i 1}}\left(m_{i-1}\right)+Q_{J_{i 2}}\left(\mu_{i+1}\right)-Q_{J_{i 3}}\left(m_{i}\right)\right)}\right) \\
& \times\left(\prod_{i=1}^{M} \frac{\bar{S}_{\rho_{i} \mu_{i}}^{k_{i}} S_{\tau_{i} \mu_{i}}^{k_{i}} S_{\mu_{i} \nu_{i}}^{k_{i}}}{S_{0 \mu_{i}}^{k_{i}}}\right)\left(\prod_{i=1}^{M-1} N_{r_{i} t_{i}^{\dagger}} \frac{{ }_{i}}{S_{d_{i} m_{i}}^{\kappa_{i+1}}} \bar{S}_{m_{i} m_{i}}^{\kappa_{i+1}} S_{m_{i} p_{i}}^{\kappa_{i+1}}\right) \widehat{\chi}_{[\nu, n, p]} \\
& =\sum_{J, d} \sum_{\substack{\mu_{i}, m_{i} \in \operatorname{Rep}(G) \\
[\nu, n, p] \in \operatorname{Rep} G}}\left(\prod_{i=1}^{M} \frac{\bar{S}_{\rho_{i} \mu_{i}}^{k_{i}} S_{\tau_{i} \mu_{i}}^{k_{i}} e^{2 \pi i Q_{J_{i-1,2}}\left(\mu_{i}\right)} S_{\mu_{i} \nu_{i}}^{k_{i}}}{S_{0 \mu_{i}}^{k_{i}}}\right) \\
& \times\left(\prod_{i=1}^{M-1} N_{r_{i} t_{i}^{\dagger}} \frac{d_{i}}{S_{d_{i} m_{i}}^{\kappa_{i+1}} e^{-2 \pi i Q_{J_{i 3}}\left(m_{i}\right)} \bar{S}_{m_{i} n_{i}}^{\kappa_{i+1}} e^{2 \pi i Q_{J_{i+1,1}}\left(m_{i}\right)} S_{m_{i} p_{i}}^{\kappa_{i+1}}} \widehat{S}_{0 m_{i}}\right) \widehat{\chi}_{[\nu, n, p]} \\
& =\sum_{J, d} \sum_{\substack{\mu_{i}, m_{i} \operatorname{Rep}(G) \\
[\nu, n, p] \in \operatorname{Rep} G}}\left(\prod_{i=1}^{M} \frac{\bar{S}_{\rho_{i} \mu_{i}}^{k_{i}} S_{\tau_{i} \mu_{i}}^{k_{i}} S_{\mu_{i} J_{i-1,2} \nu_{i}}^{k_{k}}}{S_{0 \mu_{i}}^{k_{i}}}\right) \\
& \times\left(\prod_{i=1}^{M-1} N_{r_{i} t_{i}^{\dagger}} \frac{S_{d_{i} m_{i}}^{\kappa_{i+1}} \bar{S}_{m_{i} J_{i 3} n_{i}}^{\kappa_{i+1}} S_{m_{i} J_{i+1,1} p_{i}}^{\kappa_{i+1}}}{S_{0 m_{i}}}\right) \widehat{\chi}_{[\nu, n, p]} \\
& =\sum_{J, d} \sum_{[\nu, n, p] \in \operatorname{Rep}_{G}}\left(\prod_{i=1}^{M} N_{\tau_{i} J_{i-1,2} \nu_{i}}^{\rho_{i}}\right)\left(\prod_{i=1}^{M-1} N_{r_{i}, t_{i}^{\dagger}}^{d_{i}} N_{d_{i}, J_{i+1,1} p_{i}}^{J_{i 3} n_{i}}\right) \widehat{\chi}_{[\nu, n, p]}
\end{aligned}
$$

with $J_{0,2}=J_{1,1}, J_{M, 1}=$ id. $N_{\mu \nu}{ }^{\rho}$ is the fusion coefficient. Here we have used

$$
S_{J \mu \nu}=e^{2 \pi i Q_{J}(\nu)} S_{\mu \nu}, \quad S_{\mu \nu}=S_{\nu \mu} .
$$

Obviously, the coefficients of characters are semi-positive integers. In addition, the number of identity operator $(\nu=n=p=0)$ is

$$
\begin{gathered}
\sum_{J, d}\left(\prod_{i=1}^{M} N_{\tau_{i} J_{i-1,2} 0} \rho_{i}\right)\left(\prod_{i=1}^{M-1} N_{r_{i}, t_{i}^{\dagger}}^{d_{i}} N_{d_{i}, J_{i+1,1} 0}^{J_{i 3} 0}\right) \\
=\sum_{J, d}\left(\prod_{i=1}^{M} N_{\tau_{i} 0}^{J_{i-1,2}^{-1} \rho_{i}}\right)\left(\prod_{i=1}^{M-1} N_{r_{i}, t_{i}^{\dagger}}^{d_{i}} N_{d_{i}, 0}^{J_{i+1,1}^{-1} J_{i 3} 0}\right) \\
=\sum_{J, d}\left(\prod_{i=1}^{M} \delta_{\tau_{i}}^{J_{i-1,2}^{-1} \rho_{i}}\right)\left(\prod_{i=1}^{M-1} N_{r_{i}, t_{i}^{\dagger}}^{d_{i}} \delta_{d_{i}}^{J_{i+1,1}^{-1} J_{i 3} 0}\right) \\
=\sum_{J} \prod_{i=1}^{M} \delta_{\tau_{i}}^{J_{i-1,2}^{-1} \rho_{i}} \prod_{i=1}^{M-1} N_{r_{i}, t_{i}^{\dagger}}^{J_{i+1,1}^{-1} J_{i 3} 0} .
\end{gathered}
$$


Here we have used the following properties of the fusion matrix.

$$
N_{\mu \nu}^{\rho}=N_{\mu J \nu}^{J \rho}, \quad N_{\mu 0}^{\rho}=\delta_{\mu}^{\rho}, \quad N_{\mu \nu}^{\rho}=N_{\nu \mu}^{\rho}, \quad N_{\mu \nu}^{\rho}=N_{\mu \rho^{\dagger}}^{\nu^{\dagger}} .
$$

The second factor can be transformed as

$$
\begin{aligned}
& N_{r_{i} t_{i}{ }^{+}}{ }^{J_{i+1,1}{ }^{-1} J_{i, 3} 0}=N_{r_{i}\left(J_{i+1,1}{ }^{-1} J_{i, 3} 0\right)^{+}}{ }^{t_{i}}=\sum_{\sigma_{i}} \frac{\bar{S}_{J_{i+1,1}{ }^{-1} J_{i, 3} 0, \sigma_{i}} S_{r_{i} \sigma_{i}} S_{t_{i} \sigma_{i}}}{S_{0 \sigma_{i}}} \\
& =\sum_{\sigma_{i}} e^{2 \pi i\left(Q_{J_{i+1,1}}\left(\sigma_{i}\right)-Q_{J_{i, 3}}\left(\sigma_{i}\right)\right)} \frac{\bar{S}_{0, \sigma_{i}} S_{r_{i} \sigma_{i}} S_{t_{i} \sigma_{i}}}{S_{0 \sigma_{i}}} \\
& =\sum_{\sigma_{i}} \frac{S_{J_{i+1,1} J_{i, 3}{ }^{-1} 0, \sigma_{i}} S_{r_{i} \sigma_{i}} S_{t_{i} \sigma_{i}}}{S_{0 \sigma_{i}}}=\sum_{\sigma_{i}} \frac{\bar{S}_{\left(J_{i+1,1} J_{i, 3}{ }^{-1} 0\right)^{+}, \sigma_{i}} S_{r_{i} \sigma_{i}} S_{t_{i} \sigma_{i}}}{S_{0 \sigma_{i}}} \\
& =N_{r_{i} J_{i+1,1} J_{i, 3}^{-1} 0^{t_{i}}}=N_{r_{i} 0} 0^{J_{i+1,1}{ }^{-1} J_{i, 3} t_{i}}=\delta_{r_{i}}^{J_{i+1,1}{ }^{-1} J_{i, 3} t_{i}} \\
& =\delta_{t_{i}}^{J_{i+1,1} J_{i, 3}^{-1} r_{i}} \text {. }
\end{aligned}
$$

Finally, we get

$$
Z_{(\rho, r),(\tau, t)}=\sum_{J} \prod_{i=1}^{M} \delta_{\tau_{i}}^{J_{i-1,2}^{-1} \rho_{i}} \delta_{t_{i}}^{J_{i+1,1} J_{i, 3}{ }^{-1} r_{i}}+\cdots .
$$

The dots include the contribution from the other states. In the meantime, the $|(\rho, r)\rangle$ is invariant under (4.5). Thus we conclude that the unique identity operator appears if and only if boundary states are equivalent up to $\mathcal{G}_{\text {id }}$. This completes the proof of the Cardy condition.

Open Access. This article is distributed under the terms of the Creative Commons Attribution License (CC-BY 4.0), which permits any use, distribution and reproduction in any medium, provided the original author(s) and source are credited.

\section{References}

[1] C. Nayak, M.P.A. Fisher, A.W.W. Ludwig and H.H. Lin, Resonant multilead point-contact tunneling, Phys. Rev. B 59 (1999) 15694 [cond-mat/9710305].

[2] T. Giamarchi, Quantum physics in one dimension, Oxford University Press, Oxford U.K. (2003).

[3] I. Affleck, Exact critical exponents for quantum spin chains, nonlinear $\sigma$-models at $\theta=\pi$ and the quantum Hall effect, Nucl. Phys. B 265 (1986) 409 [INSPIRE].

[4] I. Affleck and F.D.M. Haldane, Critical theory of quantum spin chains, Phys. Rev. B 36 (1987) 5291 [INSPIRE].

[5] T. Quella, I. Runkel and G.M.T. Watts, Reflection and transmission for conformal defects, JHEP 04 (2007) 095 [hep-th/0611296] [INSPIRE].

[6] T. Kimura and M. Murata, Current reflection and transmission at conformal defects: applying BCFT to transport process, Nucl. Phys. B 885 (2014) 266 [arXiv:1402.6705] [INSPIRE]. 
[7] C. Bachas, J. de Boer, R. Dijkgraaf and H. Ooguri, Permeable conformal walls and holography, JHEP 06 (2002) 027 [hep-th/0111210] [INSPIRE].

[8] E. Wong and I. Affleck, Tunneling in quantum wires: a boundary conformal field theory approach, Nucl. Phys. B 417 (1994) 403 [cond-mat/9311040].

[9] M. Oshikawa and I. Affleck, Defect lines in the Ising model and boundary states on orbifolds, Phys. Rev. Lett. 77 (1996) 2604 [hep-th/9606177] [INSPIRE].

[10] M. Oshikawa and I. Affleck, Boundary conformal field theory approach to the critical two-dimensional Ising model with a defect line, Nucl. Phys. B 495 (1997) 533 [cond-mat/9612187] [INSPIRE].

[11] T. Quella and V. Schomerus, Symmetry breaking boundary states and defect lines, JHEP 06 (2002) 028 [hep-th/0203161] [INSPIRE].

[12] A. Recknagel and V. Schomerus, Boundary conformal field theory and the worldsheet approach to D-branes, Cambridge University Press, Cambridge U.K. (2013).

[13] I. Affleck, Conformal field theory approach to the Kondo effect, Acta Phys. Polon. B 26 (1995) 1869 [cond-mat/9512099] [INSPIRE].

[14] S. Fredenhagen and T. Quella, Generalised permutation branes, JHEP 11 (2005) 004 [hep-th/0509153] [INSPIRE].

[15] I. Affleck and A.W.W. Ludwig, Universal noninteger 'ground state degeneracy' in critical quantum systems, Phys. Rev. Lett. 67 (1991) 161 [INSPIRE]. 